\title{
The Peculiarities of the Clinical Effect of Hypoxene on the Antioxidant Defense System in Patients With Chronic Obstructive Bronchitis (COB)
}

\author{
Bataev Hizir M.* Yaraskhanov Rasul R. Bataev Akhmatkhan H.
}

\author{
Medical Institute of the «Chechen State University» Grozny, Russia \\ *Corresponding author. Email: hizir62@mail.ru
}

\begin{abstract}
Today, one of the leading problems of modern health care is the problem of diagnosis and treatment of chronic obstructive bronchitis. This pathology has led to increasing rates of morbidity and mortality around the world, which are steadily leading to an increase in economic and social damage in all countries. In the nosological structure, chronic obstructive bronchitis is the leader among other pathologies in terms of the number of days of disability and the reasons for the development of disability in the population. Experts note that 3.17 million people in the world died from complications arising from the exacerbation of the disease in 2014, which accounted for almost $5 \%$ of all deaths in the world. The forecast compiled by WHO experts indicates that this nosology will soon become not only the most widespread human disease, but also become one of the leading causes of mortality. Along with this, a tendency towards a decrease in the frequency of deaths due to the phenomena of impaired coronary circulation, tumor processes, tuberculosis and other nosologies is assumed. Prospects in resolving the problem with the diagnosis and treatment of chronic obstructive bronchitis have emerged in connection with the creation and introduction of new drugs with antioxidant properties, one of which is "Hypoxen". Despite the positive antihypoxic and antioxidant effects, the mechanism of action of hypoxene in patients with $\mathrm{COB}$ is still poorly understood. Based on all that has been said above, interest in studying the effect of hypoxene on the antioxidant defense system of the body is growing every day.
\end{abstract}

\section{Keywords: chronic obstructive bronchitis, hypoxene, lipid peroxidation, antioxidant protection.}

\section{INTRODUCTION}

The issue of timely diagnosis and correct effective treatment of chronic obstructive pulmonary disease is becoming a leading problem in the modern healthcare system. The medical and social significance of this aspect is becoming more acute every day [1]. According to experts, from 4 to $15 \%$ of the world's population suffer from chronic obstructive pulmonary diseases (COPD) [2]. The results of statistical studies by the World Health Organization confirm the factor of the huge economic damage caused by COPD to the population of countries around the world [3]. Analysis data indicate that over the period from 1990 to 2020, chronic obstructive pulmonary disease, among other diseases causing significant economic damage to countries of the world, moved from 12th place to 5th place, leaving behind other pathologies of the respiratory tract [4].

COPD, as a typical lung pathology, includes three main clinical nosologies: pulmonary emphysema, bronchial asthma, chronic bronchitis [5]. Among the above pathologies, it is chronic obstructive bronchitis that is recognized as the most pronounced medical and social problem today. Treatment of a patient with chronic obstructive bronchitis is 3 times more expensive than the management of a patient with bronchial asthma [6].

Sources devoted to the problem of diagnosis and treatment of COB are very numerous. It seems that further analyzes of the current situation around this nosology may become unpromising. However, this point of view is not consistent, because indicators of the frequency and clinical component of the course of the disease, complications that often lead to disability of the population, necessitate the search for the true factors of the onset and progression of pathology [7].

An analysis of the work carried out over the past years suggests the importance of the body's antioxidant defense in the pathogenesis of COD [8]. Exacerbation of chronic obstructive bronchitis occurs with the characteristic development of an imbalance that occurs between the chains of antioxidant defense of the human body. This discrepancy lies in the fact that there is a decrease in the activity of the body's antioxidant defense system and a significant increase in the percentage and quantitative content of lipid peroxidation objects in the body. Elements formed during lipid peroxidation have a number of features associated with the fact that they are prone to prolonged modification of inflammation, exacerbation of the course of processes accompanied by the development 
of bronchospasm, and also contribute to the formation of immune deficiency [9].

An alternative solution to the problem related to the diagnosis and treatment of chronic obstructive bronchitis is the introduction of new drugs that have a pronounced tendency to antioxidant effect. Hypoxen considered in this article is in the list of these drugs. The data of experiments carried out in order to reveal the clinical effect of this category of drugs under artificially created conditions of hypoxia showed that there is a qualitative decrease in tissue demand for oxygen, due to its best capture and assimilation by cells [10]. Based on this, the therapy will be more successful, if antioxidants are used along with the appointment of pathogenetic treatment methods. Despite the proven antihypoxic and antioxidant effects, the mechanism of action of hypoxene on the antioxidant defense system in patients with $\mathrm{COB}$ remains insufficiently studied to date.

Based on all that has been said above, it follows that the interest arising in the study of the nature of the effect of hypoxene on the clinical and functional indicators and processes of the antioxidant defense system of the body in patients with chronic obstructive bronchitis is getting stronger every day.

\section{METHODS AND MATERIALS}

In the course of the study, a clinical observation was organized with the participation of 75 patients (40 men and 35 women) aged 35 to 75 years who sought medical help at an inpatient medical institution due to exacerbation of chronic obstructive bronchitis, as well as a group of people aged 35-75 years leading a healthy lifestyle, in the amount of 25 people.

The selection of patients for participation in the analysis was carried out according to the following parameters: the consent of the patient to participate in the ongoing clinical observation, the presence of a clinically confirmed diagnosis of chronic obstructive bronchitis in the patient, strict adherence to all the prescriptions of medical personnel. Patients were excluded from the study if the following conditions were present: inconsistency in the age cohort (if the subject is older than 75 years old or younger than 35 years old), undesired to take part in the study, lack of adherence to the requirements of the medical community, individual intolerance to drugs used in clinical analysis; the presence of concomitant chronic diseases in patients.

In all analyzed cases, the diagnosis "Chronic obstructive bronchitis" was made on the basis of the clinical picture, the data of X-ray and bronchoscopic studies, as well as the results of functional laboratory procedures. Respiratory function was assessed using a polyspirometer coupled with a special software, according to the following series of parameters: Tiffeneau index, peak volumetric expiratory flow rate, lung capacity, forced expiratory volume in 1 second.

The contingent of people suffering from chronic obstructive bronchitis was distributed according to the severity in accordance with the established recommendations of "GOLD-2019" ("Global Initiative for Chronic Obstructive Pulmonary Disease"), taking into account the data of the forced expiratory volume in 1 second (FEV1) in relation to the reference values: mild degree corresponds to FEV1> $80 \%$ of the reference values; moderate severity includes values from a range of $50 \%<$ FEV1 < 80\%; severe cases mean FEV1 <50 \%

The patient examination plan also included an analysis of the state of antioxidant protection. It was carried out with a consistent study of such indicators as: total antioxidant activity of blood plasma, catalase content, the value of superoxide dismutase activity, indicators of the content of diene conjugates and the concentration of malondialdehyde in the blood serum. Using the method of E.B. Spektor, the index of the total antioxidant activity was calculated. The catalase content was calculated from the data obtained during the reaction of hydrogen peroxide and ammonium molybdate. Superoxide dismutase value was determined using reagents on an automatic analyzer. The character of diene conjugates in the blood was determined by the method of V.B. Gavrilov. The determination of the amount of malonic dialdehyde was carried out according to the reaction with the participation of thiobarbituric acid.

The presence and severity of the main symptoms accompanying the clinical course of chronic obstructive bronchitis in the analyzed groups were assessed after 3, 5, $7,10,13,15,18,20$ days from the start of therapy. The interpretation of the severity of symptoms was carried out according to the following system (Table 1). When summing these points, the total was displayed. The indicators of the activity of antioxidant protection were investigated upon admission to a medical institution and upon discharge from it.

Table 1 Assessment of the severity of clinical symptoms of chronic obstructive bronchitis

\begin{tabular}{|l|c|c|c|c|}
\hline \multirow{2}{*}{$\begin{array}{c}\text { Clinical } \\
\text { Symptoms }\end{array}$} & \multicolumn{4}{|c|}{ Points } \\
\cline { 2 - 5 } & $\mathbf{0}$ points & 1 point & 2 points & 3 points \\
\hline Cough & - & $\begin{array}{c}\text { intermittent cough in the } \\
\text { morning }\end{array}$ & $\begin{array}{c}\text { persistent cough in the } \\
\text { morning }\end{array}$ & $\begin{array}{c}\text { persistent cough all } \\
\text { day long }\end{array}$ \\
\hline Sputum & - & $\begin{array}{c}\text { a small amount of mucous } \\
\text { sputum }\end{array}$ & $\begin{array}{c}\text { profuse mucous or } \\
\text { mucopurulent sputum }\end{array}$ & $\begin{array}{c}\text { a large amount of } \\
\text { purulent sputum }\end{array}$ \\
\hline Dyspnea & - & $\begin{array}{c}\text { shortness of breath when } \\
\text { walking fast }\end{array}$ & $\begin{array}{c}\text { shortness of breath when } \\
\text { walking up to 100 m }\end{array}$ & dyspnea at rest \\
\end{tabular}


The main starting point of the study was the distribution of patients into two groups. 1) The main group: 48 patients who received the drug "Hypoxen" in combination with basic therapy. "Hypoxen" was prescribed in tablet form with a dosage of $250 \mathrm{mg} 3$ times a day with meals for 2-2.5 weeks in a hospital with a further extension of the drug intake at the outpatient stage of the study for a period of 1.5-2 weeks. The duration of the therapy was 1 month. 2) Comparison group: 27 patients who received basic therapy drugs according to the GOLD recommendations (2019). Basic therapy included the following drugs and procedures: bronchodilators (Atrovent in the form of inhalations by means of a nebulizer, with a dosage of 1 breath 3 times a day), expectorant drugs (Ambroxol in tablet form with a dosage of $30 \mathrm{mg} 3$ times a day) day; Bromhexine in tablet form with a dosage of $16 \mathrm{mg} 3$ times a day), antibiotics, physiotherapeutic procedures and breathing exercises. In the case of a predominance of an insignificant clinical effect, the drug Berotek was added to the above drugs. Similarly, depending on the shown therapeutic effect, additionally the therapy included Theophylline (prolonged forms), Euphyllin ( $2.4 \%$ solution of $10 \mathrm{ml}$ intravenously). Antibiotics were added to the list of drugs for treatment only on the basis of strict indications for use: analysis of the results of bacteriological sputum culture, as well as the sensitivity of microflora.

As for the statistical processing of the data obtained in the course of the work, it was carried out using the statistical software packages Microsoft Excel and Biostat

on a personal computer. With a normal distribution of values, such statistical indicators were used as: the value of the arithmetic mean (M), the error rate of the arithmetic mean $(\mathrm{m})$ with further verification of the reliability of the discrepancies according to the Student's t-test. In the case of a small number of observations and with a nonparametric form of distribution, the following methods of statistical processing of information were used to determine the confidence indicator of discrepancies: the main statistical Mann-Whitney test and the Wilcoxon statistical test. The Mann-Whitney test was used for the unrelated nature of the sample phenomena. And the Wilcoxon criterion was used with the associated nature of the sample information. Spearman's correlation analysis was also carried out in order to determine the strength of the connection between the analyzed features. Differences or correlation were considered significant at $\mathrm{P}<0.05$.

\section{RESULTS}

The data obtained as a result of the study showed the following picture.

Patients with a mild degree of chronic obstructive bronchitis upon admission to the hospital had a confirmed increase in the values of diene conjugates $(\mathrm{P}<0.05)$ in the analyzes taken in patients of both analyzed groups: the main group - 2.14 \pm 0.15 , the comparison group $2.11 \pm 0.15$ (Table 2)

Table 2 Changes in the level of indicators of the antioxidant defense system in patients with mild chronic obstructive bronchitis according to the results of the treatment

\begin{tabular}{|l|c|c|c|c|c|}
\hline \multicolumn{1}{|c|}{ Indicator } & \multirow{2}{*}{$\begin{array}{c}\text { Healthy } \\
(\mathbf{n = 2 5})\end{array}$} & \multicolumn{2}{c|}{ Main group (n= 8) } & \multicolumn{2}{c|}{ Comparison group (n = 5) } \\
\cline { 3 - 6 } & & before therapy & after therapy & before therapy & after therapy \\
\hline TAN [mmol/l] & $4.95 \pm 0.15$ & $4.12 \pm 0.18$ & $4.91 \pm 0.17$ & $4.15 \pm 0.18$ & $4.65 \pm 0.16$ \\
\hline $\mathrm{MDA}[\mathrm{nmol} / \mathrm{ms} \mathrm{s}]$ & $8.4 \pm 2.1$ & $12.3 \pm 2.3$ & $8.2 \pm 2.3$ & $12.5 \pm 2.3$ & $8.4 \pm 2.2$ \\
\hline $\mathrm{DC}[\mathrm{D} 232 / \mathrm{ml}]$ & $1.72 \pm 0.06$ & $2.14 \pm 0.15^{*}$ & $1.71 \pm 0.06$ & $2.11 \pm 0.15^{*}$ & $1.79 \pm 0.08$ \\
\hline Cat, $[\Delta \mathrm{E} 240 / \mathrm{min}]$ & $0.31 \pm 0.03$ & $0.32 \pm 0.03$ & $0.34 \pm 0.06$ & $0.32 \pm 0.06$ & $0.32 \pm 0.04$ \\
\hline SOD[a.u./mg of protein] & $1.24 \pm 0.23$ & $1.15 \pm 0.17$ & $1.23 \pm 0.17$ & $1.15 \pm 0.18$ & $1.22 \pm 0.15$ \\
\hline
\end{tabular}

Note: $*$ - reliability indicator, reflecting the degree of discrepancy between the analyzed groups and the group of healthy people; ** - reliability indicator, reflecting the degree of discrepancy between the main group and the comparison group.

The level of malondialdehyde values in patients did not increase so much $(\mathrm{P}<0.02)(12.3 \pm 2.3$ in the main group and $12.5 \pm 2.3$ in the comparison group). The values of antioxidant protection in the contingent of persons of both groups tended to decrease $(\mathrm{P}>0.05)$ : in the patients of the main group, the activity of catalase was $0.32 \pm 0.03$, that of superoxide dismutase was $1.15 \pm 0.17$, the level of total antioxidant activity was $4.12 \pm 0.18$; in the patients of the comparison group the level of catalase activity was
$0.32 \pm 0.06$, that of superoxide dismutase was $1.15 \pm 0.18$, the level of general antioxidant activity was $4.15 \pm 0.18$. Against the background of the therapy in the studied patients of both groups, a confirmed decrease in the data on the activity of lipid peroxidation was recorded. In the contingent of persons who received "Hypoxen" as part of therapy, at the stage of discharge from the hospital, the content of diene conjugates was $1.71 \pm 0.06$, and the content of malondialdehyde was $8.2 \pm 2.3$. And in patients 
undergoing standard therapy, the content of diene conjugates was $1.79 \pm 0.08$ and that of malondialdehyde was $8.4 \pm 2.2$. The difference in the indicators in the study groups was insignificant $(\mathrm{P}>0.05)$. These antioxidant defense systems in the analyzed patients of both groups, as a result of the therapy, were characterized by a tendency to increase up to the normative indicators: the level of catalase values in patients receiving "Hypoxen" was $0.34 \pm 0.06$, and the level of superoxide dismutase was $1.23 \pm 0.17$. And in patients undergoing standard therapy, catalase level reached $0.32 \pm 0.04$, and that of superoxide dismutase was $1.22 \pm 0.15$. The values of the total antioxidant activity in the contingent of individuals in both groups also increased: in the main group to $4.91 \pm 0.17$, and to $4.65 \pm 0.16$ in the comparison group.

Patients with an average degree of chronic obstructive bronchitis upon admission to a medical institution due to an exacerbation of the existing pathology demonstrated an established increase in the parameters of lipid peroxidation processes in the analyzes taken (Table 3 ).

Table 3 Changes in the level of indicators of the antioxidant defense system in patients with moderate severity of chronic obstructive bronchitis according to the results of the treatment

\begin{tabular}{|l|c|c|c|c|c|}
\hline \multicolumn{1}{|c|}{ Indicator } & \multirow{2}{*}{$\begin{array}{c}\text { Healthy } \\
(\mathbf{n}=\mathbf{2 5})\end{array}$} & \multicolumn{2}{c|}{ Main group (n= 28) } & \multicolumn{2}{c|}{ Comparison group (n = 13) } \\
\cline { 3 - 5 } & & before therapy & after therapy & before therapy & after therapy \\
\hline TAN $[\mathrm{mmol} / \mathrm{l}]$ & $4.95 \pm 0.15$ & $3.41 \pm 0.13^{*}$ & $4.87 \pm 0.11^{* *}$ & $3.39 \pm 0.12^{*}$ & $3.94 \pm 0.23$ \\
\hline MDA $[\mathrm{nmol} / \mathrm{ms} \mathrm{s}]$ & $8.4 \pm 2.1$ & $16.4 \pm 2.6^{*}$ & $8.7 \pm 2.2^{* *}$ & $16.3 \pm 2.7 *$ & $13.6 \pm 2.4$ \\
\hline DC $[\mathrm{D} 232 / \mathrm{ml}]$ & $1.72 \pm 0.06$ & $3.26 \pm 0.18^{*}$ & $1.76 \pm 0.04^{* *}$ & $3.31 \pm 0.17^{*}$ & $2.61 \pm 0.16$ \\
\hline Cat, $[\Delta \mathrm{E} 240 / \mathrm{min}]$ & $0.31 \pm 0.03$ & $0.20 \pm 0.03^{*}$ & $0.34 \pm 0.05^{* *}$ & $0.22 \pm 0.04^{*}$ & $0.26 \pm 0.06$ \\
\hline SOD [a.u./mg of protein] & $1.24 \pm 0.23$ & $0.95 \pm 0.14^{*}$ & $1.22 \pm 0.14^{* *}$ & $0.97 \pm 0.11^{*}$ & $1.03 \pm 0.17$ \\
\end{tabular}

Note: * - reliability indicator, reflecting the degree of discrepancy between the analyzed groups and the group of healthy people;

** - reliability indicator, reflecting the degree of discrepancy between the main group and the comparison group.

Among the representatives of the main group, the indicators of diene conjugates during hospitalization in a medical institution were $3.26 \pm 0.18$, and those of malondialdehyde were 16.4 \pm 2.6 . And in the representatives of the comparison group, the values of diene conjugates were $3.31 \pm 0.17$, and for malondialdehyde the values were $16.3 \pm 2.7$. The discrepancy between the indicators of sick people and those of healthy people was significantly reflected $(\mathrm{P}<0.05)$. The antioxidant system of the contingent of persons suffering from chronic obstructive bronchitis of moderate severity was characterized by a significant decrease in catalase, superoxide dismutase and the level of antioxidant activity of blood plasma. Representatives of the main group had the following indicators: $0.20 \pm 0.03$ of catalase, $0.95 \pm 0.14$ of superoxide dismutase, antioxidant activity was $3.41 \pm 0.13$. And patients from the comparison group had the following results: $0.22 \pm 0.04$ of catalase, $0.97 \pm 0.11$ of superoxide dismutase and antioxidant activity was $3.39 \pm 0.12$. The introduction of hypoxene into the therapy for the contingent of patients with moderate severity of chronic obstructive bronchitis had the effect of a significant decrease in the activity of lipid peroxidation processes. At the time of discharge from the hospital, the indicators of diene conjugates and malondialdehyde in the main group were $1.76 \pm 0.04(\mathrm{P}<0.05)$ and $8.7 \pm 2.2$ $(\mathrm{P}<0.05)$, respectively. As for the patients who received standard therapy, the indices for diene conjugates and malondialdehyde were $2.61 \pm 0.16(\mathrm{P}>0.05)$ and $13.6 \pm 2.4$ $(\mathrm{P}>0.05)$, respectively. In the period of completion of the treatment in both groups of patients, an increase in the value of the total antioxidant activity of blood plasma was recorded. However, a significant increase in indicators was found in patients who received hypoxen as part of the therapy: $4.87 \pm 0.11$ in the main group and $3.94 \pm 0.23$ in the comparison group. A similar situation occurred in the dynamic observation of the content of catalase and superoxide dismutase (1st group: $0.34 \pm 0.05$ of catalase ( $\mathrm{P}<0.05)$, and $1.22 \pm 0.14$ of superoxide dismutase ( $\mathrm{P}<0.05)$; 2nd group: $0.26 \pm 0.06$ of catalase $(\mathrm{P}>0.05)$, and $1.03 \pm 0.17$ of superoxide dismutase $(\mathrm{P}>0.05)$ ).

The initial state of the antioxidant defense system in patients with severe chronic obstructive bronchitis had characteristic features: a more pronounced $(\mathrm{P}<0.05)$ increase in the content of lipid peroxidation products against the general background of a decrease in the activity of the antioxidant defense system. Indicators of diene conjugates and malondialdehyde during hospitalization in a medical institution in the main group were $4.34 \pm 0.16$ and $18.5 \pm 2.7$, respectively. And in patients from the comparison group, those values were $4.32 \pm 0.18$ and $18.3 \pm 2.8$, correspondingly (Table 4). The antioxidant system in the representatives of the main group had the following indicators: $0.15 \pm 0.03$ of catalase, $0.84 \pm 0.12$ of superoxide dismutase, the indicators of antioxidant activity were $2.83 \pm 0.12$. And the patients from the comparison group had the following results: 
$0.16 \pm 0.02$ of catalase, $0.82 \pm 0.13$ of superoxide dismutase and the antioxidant activity indices were $2.81 \pm 0.12$. When evaluating the indicators after the treatment in the contingent of patients from both groups, a tendency towards a decrease in the activity of lipid peroxidation processes was recorded, but the levels of indicators did not reach the standard values. A decrease in the activity of lipid peroxidation processes was more clearly seen in patients who received additional hypoxene. The values of indicators at discharge from a hospital in patients of group 1 were: $2.15 \pm 0.02$ of diene conjugates $(\mathrm{P}<0.05), 10.5 \pm 2.3$ of malondialdehyde $(\mathrm{P}<0.05)$. In patients of the second group, the following results were obtained: the level of diene conjugates was $3.01 \pm 0.16 \quad(\mathrm{P}<0.05)$, that of malondialdehyde was $15.4 \pm 2.4(\mathrm{P}<0.05)$. When analyzing the results of the state of the antioxidant defense system at the time of discharge from the medical institution, a tendency to increase its activity was recorded in both

Table 4 Changes in the level of indicators of the antioxidant defense system in patients with severe chronic obstructive bronchitis according to the results of the treatment

\begin{tabular}{|c|c|c|c|c|c|}
\hline \multirow[t]{2}{*}{ Indicator } & \multirow{2}{*}{$\begin{array}{l}\text { Healthy } \\
(n=25)\end{array}$} & \multicolumn{2}{|c|}{ Main group $(n=12)$} & \multicolumn{2}{|c|}{ Comparison group $(\mathbf{n}=9)$} \\
\hline & & before therapy & after therapy & before therapy & after therapy \\
\hline TAN [mmol/l] & $4.95 \pm 0.15$ & $2.83 \pm 0.12 *$ & $3.49 \pm 0.13 * *$ & $2.81 \pm 0.12 *$ & $3.11 \pm 0.22$ \\
\hline $\mathrm{MDA}[\mathrm{nmol} / \mathrm{ms} \cdot \mathrm{s}]$ & $8.4 \pm 2.1$ & $18.5 \pm 2.7 *$ & $10.5 \pm 2.3 * *$ & $18.3 \pm 2.8 *$ & $15.4 \pm 2.4$ \\
\hline $\mathrm{DC}[\mathrm{D} 232 / \mathrm{ml}]$ & $1.72 \pm 0.06$ & $4.34 \pm 0.16^{*}$ & $2.15 \pm 0.02 * *$ & $4.32 \pm 0.18^{*}$ & $3.01 \pm 0.16$ \\
\hline Cat, $[\Delta \mathrm{E} 240 / \mathrm{min}]$ & $0.31 \pm 0.03$ & $0.15 \pm 0.03 *$ & $0.36 \pm 0.07 * *$ & $0.16 \pm 0.02 *$ & $0.27 \pm 0.06$ \\
\hline SOD [a.u./mg of protein] & $1.24 \pm 0.23$ & $0.84 \pm 0.12 *$ & $1.09 \pm 0.14 * *$ & $0.82 \pm 0.13^{*}$ & $0.91 \pm 0.16$ \\
\hline
\end{tabular}

Note: * - reliability indicator, reflecting the degree of discrepancy between the analyzed groups and the group of healthy people;

** - reliability indicator, reflecting the degree of discrepancy between the main group and the comparison group.

We get the following conclusions. The introduction of hypoxene into the therapy for patients with exacerbation of chronic obstructive bronchitis has a beneficial effect on the body's antioxidant defense system. In contrast to patients who received only standard therapy. Moreover, a more pronounced clinical effect is observed in persons with an average degree of chronic obstructive bronchitis.

\section{CONCLUSION}

1. The introduction of the drug "Hypoxen", which has proven antioxidant and antihypoxant effects, in the composition of the therapy for chronic obstructive bronchitis leads to the development of a positive effect on the course of the disease, largely in the presence of moderate severity of this pathology.

2. Appointment of hypoxene to patients with exacerbation of chronic obstructive bronchitis fully meets the requirements of pathogenetic therapy due to the presence of fragmentation of interaction chains in the work groups. A more striking clinical effect was also obtained in the main group. The indicator of the general antioxidant activity was $3.49 \pm 0.13$. And in the second group, the indicator reached the level of $3.11 \pm 0.22$. This trend was also noted in the dynamics of catalase and superoxide dismutase indicators. In patients of the main group, the level of values showed the following data: $0.36 \pm 0.07$ of catalase $(\mathrm{P}<0.05)$, $1.09 \pm 0.14$ of superoxide dismutase $(\mathrm{P}<0.05)$. In patients of the second group, the level of values showed the following data: $0.27 \pm 0.06$ of catalase $(P>0.05), 0.91 \pm 0.16$ of superoxide dismutase $(\mathrm{P}>0.05)$. From the above results, it follows that in patients with severe chronic obstructive bronchitis, clinical remission is established without normalization of lipid peroxidation parameters and the antioxidant defense system, which, apparently, is associated with the activity of the inflammation process in the respiratory tract. 
[2] E.I. Shmelev, COPD and problems, 2016, p. 43.

[3] M.V. Beltyukova, Chronic obstructive pulmonary disease, 2014, p. 6.

[4] N.R. Anthonisen, COPD is a global problem, 2012, p. 15.

[5] Z.R. Aisanov, The main forms of obstructive pulmonary disease, 2011, p. 23.

[6] P.J. Barnes, Clinical Therapy and Physician Tactics for COPD, 2006, p. 83.

[7] A.G. Chuchalin, Chronic obstructive pulmonary disease and concomitant diseases, Media Sphere 8 (2013) 41.

[8] A.S. Sokolov, A.G. Chuchalin, V.G. Novozhenov, Management of patients with diseases of the respiratory system, 1996, $125 \mathrm{p}$.
[9] M.Sh. Dadaev, The state of lipid peroxidation processes in patients with chronic obstructive bronchitis with various degrees of severity, Actual issues of modern medicine, Abstracts of the 80th Anniversary All-Russian Baikal scientific-practical conference of young scientists and students with international participation, Irkutsk, 2013, pp. 336-338.

[10] H.M. Bataev, M.Sh. Dadaev, M.D. Shikhnabieva, Research methods for diseases of the respiratory system, Textbook, Grozny, 2012, p. 13.

[11] H.M. Bataev, The use of hypoxene in the complex treatment of patients with chronic obstructive bronchitis of moderate severity, II annual summarizing conference of the faculty of the Chechen State University, Grozny, 16 February 2013, pp. 131-132. 